\title{
Placement of Totally Implantable Venous Access Ports Using the Single-Incision Technique: Outcomes and Complications
}

\author{
Seo Jin Jang ${ }^{1}$, Jae Hyun Kwon ${ }^{1,{ }^{*}}$, Yoon Ki Cha ${ }^{1}$ and Do Yeun $\mathrm{Kim}^{2}$ \\ ${ }^{1}$ Department of Radiology, Dongguk University Ilsan Hospital, Dongguk University School of Medicine, Goyang, South Korea \\ ${ }^{2}$ Department of Internal Medicine, Dongguk University Ilsan Hospital, Dongguk University College of Medicine, Goyang, South Korea \\ "Corresponding author: Department of Radiology, Dongguk University Ilsan Hospital, Dongguk University School of Medicine, Goyang, South Korea, Tel: +82-31-961-7828, Email: \\ jhkwon17@naver.com \\ Received 2020 February 15; Accepted 2020 July 11.
}

\begin{abstract}
Background: A totally implantable venous access port (TIVAP) is an essential component of care for oncology patients. Conventional placement of the TIVAP is performed through the internal jugular vein or the subclavian vein using a tunneled catheter, which involves creating two incisions. However, the conventional technique has several potential limitations. To address these limitations, a single-incision technique without a second incision or subcutaneous tunneling has been extensively tested since first being introduced by Glenn in 2007.

Objectives: The purpose of this study was to evaluate the technical success, clinical outcomes, and complications of the singleincision technique for the placement of TIVAPs.

Patients and Methods: Between January 2013 and June 2017,182 TIVAPs were placed by a single-incision technique in 175 patients, including 79 men and 96 women (mean age, 62.4 years; range: 20 - 88 years). Electronic medical records were retrospectively reviewed to obtain patient data, outcomes, and complication rates.

Results: A total of 40,594 catheter maintenance days (median, 221.9 days; range, 1 - 889 days) were recorded for 182 TIVAPs in 176 patients. Technical and clinical success rates were both $100 \%$. A total of 25 complications (complication rate, 13.74\%) occurred, including catheter occlusion (5.49\%), catheter-related infection (5.49\%), wound dehiscence (1.10\%), catheter kinking (0.55\%), venous thrombosis (0.55\%), and extravasation during infusion (0.55\%).

Conclusion: The single-incision technique for TIVAP via the axillary vein was safe and efficient with high technical and clinical success rates. This new technique may be a good alternative to conventional techniques.
\end{abstract}

Keywords: Central Venous Access, Venous Access Ports, Catheters, Axillary Vein, Complications

\section{Background}

A totally implantable venous access port (TIVAP) has become an essential tool for patients who require longterm venous access (1-4). Conventionally, placement of the TIVAP is performed through the internal jugular vein (IJV) or the subclavian vein by creating a tunnel between the venipuncture site and port pocket in the infraclavicular area. However, the conventional technique has several potential limitations associated with the creation of two incisions, such as a risk of bleeding, patient dissatisfaction associated with discomfort and esthetic disadvantages (5, 6). To overcome these limitations, the single-incision technique for placing TIVAPs has been extensively tested since 2007 (4-8).

\section{Objectives}

The purpose of this study was to evaluate the technical success, clinical outcomes, and complications of the single-incision technique for the placement of TIVAP.

\section{Patients and Methods}

\subsection{Patients}

The institutional review board of our institution approved this retrospective study protocol. The requirement for written informed consent was waived due to the retrospective nature of the study. Electronic medical records and radiographic images of TIVAPs performed from January 2013 to June 2017 were retrospectively reviewed. Patients who underwent placement of a TIVAP by singleincision technique via the axillary vein were included in 
this study. Exclusion criteria were: (1) placement of the TIVAP by the conventional technique and (2) placement of the TIVAP via peripheral veins in the upper extremity.

\subsection{Technique}

All procedures were performed by two interventional radiologists (JH Kwon and YH Han) who had 12 and 15 years of clinical experience in interventional radiology, respectively. The following types of ports were used: 6.5-Fr Celsite Discreet (B. Braun Medical, Boulogne Cedex, France), 8.5Fr Districath (Districlass Medical SA, Saint Etienne, France), and 8-Fr PowerPort (Bard Access System, West Salt Lake City, UT, USA).

Before the procedure, written informed consent was obtained from all patients. The right axillary vein was primarily used. Under local anesthesia, an oblique incision was made for the pocket in the infraclavicular area, and the port pocket was created by blunt dissection of subcutaneous tissue. Under ultrasound guidance, the axillary vein was punctured with a 7-cm x 21-G micropuncture needle (MAK Mini Access Kit, Merit Medical System, South Jordan, UT, USA). The needle tip was advanced diagonally upward to the axillary vein. The target vascular entry site of the puncture needle was lateral to the lateral margin of the first rib, which reduced the risk of pinch-off syndrome. An 0.018-inch hairy guidewire was inserted through the puncture needle, followed by a $5-\mathrm{Fr} \times 10-\mathrm{cm}$ coaxial introducer. A 0.035-inch guidewire was then advanced through a 5-Fr introducer to the superior vena cava (SVC) and into the inferior vena cava, thereby confirming venous access. A peel-away sheath was inserted into the axillary vein. The port catheter was introduced through a peel-away sheath, placing the catheter tip at the junction of the right atrium and SVC or within the cephalic portion of the right atrium. The TIVAP chamber connected to the catheter tip was implanted in the port pocket. Port patency was confirmed by aspiration of blood and non-problematic flushing with normal saline. A final fluoroscopic image verified the correct positioning of the catheter tip and the course of the catheter (Figure 1). Heparin solution (100 units $/ \mathrm{mL}$ ) was locked into the port chamber and catheter lumen. Postprocedural chest radiography was performed to evaluate the port, catheter, and any complications related to the procedure.

\subsection{Data Collection}

Demographic information, underlying disease, indication of port placement, history of medication, preprocedural laboratory results, target vessel, technical success, clinical success, and complications were evaluated (9, 10).

\subsection{Definitions}

Definitions used in this study followed the Society of Interventional Radiology Guidelines $(9,10)$. Technical success was defined as implantation of the TIVAP into the venous system via the axillary vein, with the tip placed at the junction of the right atrium and SVC for adequate catheter function. Clinical success was defined as the correct functioning of the port, based on the indications $(9,10)$. The total access site service interval was defined as the duration of the single access site from the day of port insertion to the day of the study endpoint. The initial device service interval was defined as the period from the day of port insertion to the day of the first revision, removal at the completion of therapy, patient death, or study endpoint. The secondary device service interval was defined as the period from the day of the first revision to the study endpoint (9, 10). The study endpoint was defined as the date of the last visit to the hospital, the date of death in electronic medical records, or the date of TIVAP removal. Revision of the device was defined as device replacement or repair without a change of access site. In accordance with Society of Interventional Radiology guidelines, complications were classified into three categories based on the timing of onset: periprocedural ( $\leq 24 \mathrm{~h}$ ), early ( $\leq 30$ days), and late ( $>30$ days); moreover, complications were graded as minor or major $(9,10)$. Complications requiring therapy, minor hospitalization, or prolonged hospitalization $(>48 \mathrm{~h})$, as well as those that resulted in an unplanned increase in the severity of the patient's condition, permanent adverse sequelae, and/or death were considered major complications $(9,10)$.

Catheter-related infections were classified as central line-associated bloodstream infection (CLABSI), catheterrelated bloodstream infection (CRBSI), and pocket infection. CLABSI was defined as a primary bloodstream infection in a patient who had developed a bloodstream infection related to an infection at another site within the previous $48 \mathrm{~h}$ period without an alternative cause (10-13). CRBSI was defined as follows: bacteremia or fungemia in a patient who had an intravascular device and one positive blood culture result obtained from the peripheral vein, clinical manifestations of infection and no apparent source for bloodstream infection. Patients with CRBSI met one of the following criteria: a positive result of semiquantitative ( $>15$ colony-forming units [cfu] per catheter segment) or quantitative ( $>10^{2}$ cfu per catheter segment) catheter culture, whereby the same organism was isolated from a catheter segment and a peripheral blood culture; and/or simultaneous quantitative cultures of blood with a ratio of $>3: 1 \mathrm{cfu} / \mathrm{mL}$ of blood (catheter vs. peripheral blood) $(12,13)$. Pocket infection was diagnosed in patients who had clinical manifestations of tenderness, erythema, induration, and/or purulence within $2 \mathrm{~cm}$ of the catheter within/overlying tissue of the port pocket. When pocket in- 

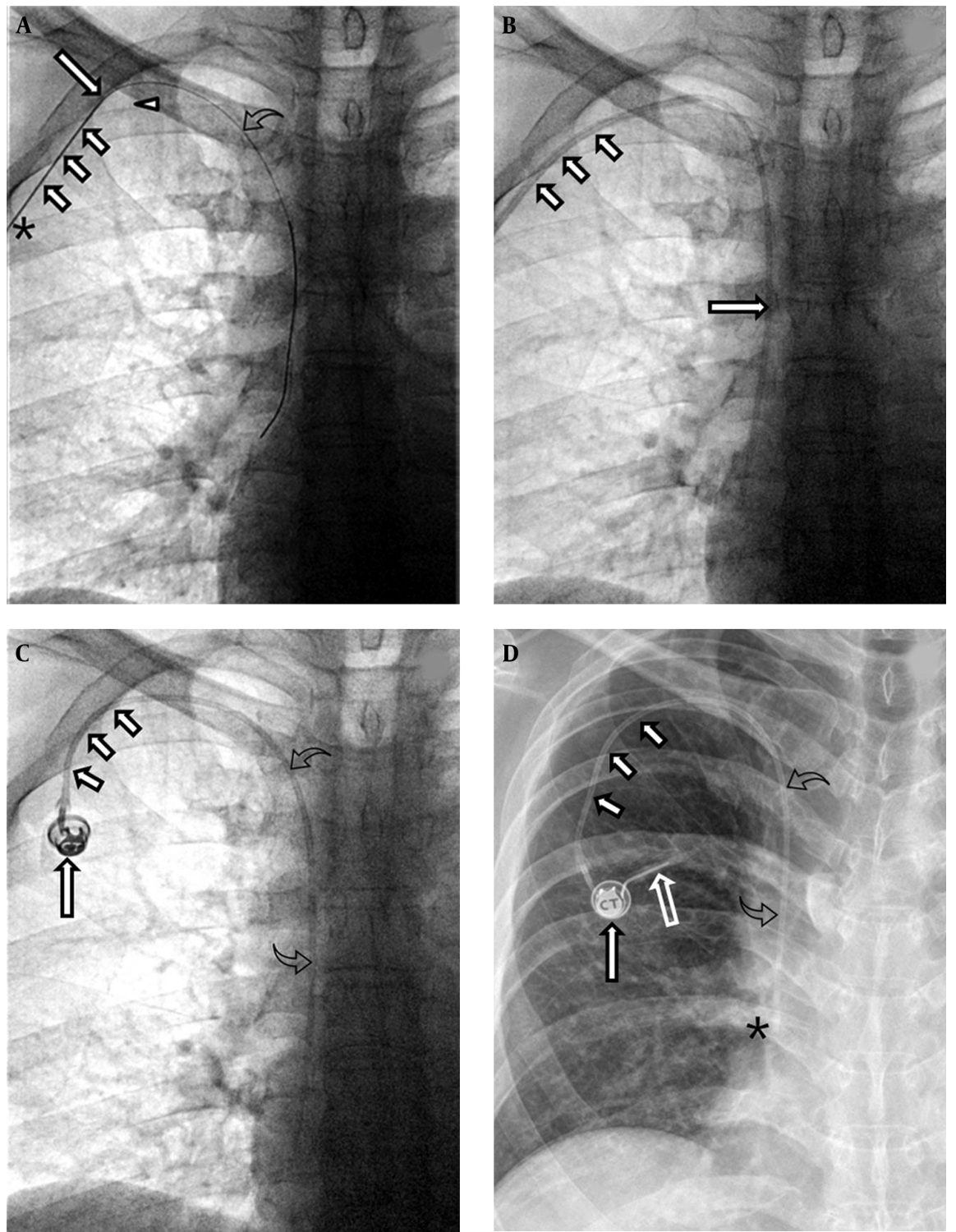

Figure 1. A 68-year-old male with a gastric lymphoma. A. Fluoroscopy reveals the tip (long arrow) and body (small arrows) of the 21-G Chiba needle. The tip of the needle is located in the right axillary vein and the body in the subcutaneous tissue of the chest wall near the skin access site (asterisk). The vascular entry site lies lateral to the lateral margin of the first rib (arrowhead). A hairy guidewire (curved arrow) was inserted through the puncture needle and the tip of the wire placed at the junction of the right atrium and superior vena cava. B. Fluoroscopy reveals the catheter (long arrow) in the superior vena cava and the peel-away sheath (small arrows) in the subcutaneous tunnel. C. Fluoroscopy reveals the venous access port (long arrow), the catheter (small arrows) in the subcutaneous tunnel, and the catheter (curved arrows) within the right brachiocephalic vein and the superior vena cava. D. A chest radiograph obtained just after port insertion reveals the venous access port (long arrow), the catheter (small arrows) in the subcutaneous tunnel, and the catheter (curved arrows) within the right brachiocephalic vein and superior vena cava. The tip of the port catheter lies at the junction of the superior vena cava and right atrium (asterisk). Note that a Huber noncoring needle (empty arrow) was inserted into the septum of the port.

fection was suspected, swab cultures were obtained from the pocket wound $(10,13)$.

\section{Results}

A total of 289 TIVAPs were implanted in 277 patients, including 182 TIVAPs implanted in 176 patients by the single incision technique, 103 TIVAPs implanted in 97 patients by the conventional technique, and four TIVAPs implanted in four patients via peripheral veins (right great saphenous, right basilic, left basilic, and right brachial veins). The demographics and underlying diseases of these 176 patients are shown in Tables 1 and 2. The numbers of ports used were as follows: 134 Celsite Discreet ports, 46 Districath 
ports, and two PowerPort ports. Technical and clinical success rates of the single incision technique were both $100 \%$ $(182 / 182)$. At the study endpoint, 42 of 182 TIVAPs were removed, 53 were in patients who had died, and the remaining 87 TIVAPs were in outpatients or inpatients. The causes of removal of 42 ports in 40 patients are described in Table 3.

\begin{tabular}{|c|c|}
\hline Variables & Values \\
\hline Age, mean (range), years & $62.4(20-88)$ \\
\hline \multicolumn{2}{|l|}{ Sex, No. (\%) } \\
\hline Male & $79(44.6)$ \\
\hline Female & $96(55.4)$ \\
\hline Height, mean (range), cm & $158.7(152.2-166.5)$ \\
\hline Weight, mean (range), kg & $55.7(48.6-64.7)$ \\
\hline PT-INR, mean (range) & $1.1(1.0-1.1)$ \\
\hline aPTT, mean (range), sec & $32(29.6-36.5)$ \\
\hline Platelet, mean (range), $\times 10^{3} \mu \mathrm{L}$ & $242(172-320)$ \\
\hline \multicolumn{2}{|l|}{ History of medication, No. (\%) } \\
\hline Antibiotics & 39 \\
\hline Antithrombotics & 5 \\
\hline Anticoagulation & 8 \\
\hline None & 130 \\
\hline \multicolumn{2}{|l|}{ Indication of port insertion, No. (\%) } \\
\hline Chemotherapy & $177(97.2)$ \\
\hline IV fluid infusion & $5(2.8)$ \\
\hline \multicolumn{2}{|l|}{ Target vessel, No. (\%) } \\
\hline Right axillary vein & $143(78.6)$ \\
\hline Left axillary vein & $39(21.4)$ \\
\hline
\end{tabular}

The total access site service interval was 40,594 days (mean, 223.04 days; range: 1 - 889 days) in 182 TIVAPs. The initial device service interval was 39,914 days (mean, 219.31 days; range: 1 - 889 days). The secondary device service interval was 679 days (mean, 67.9 days; range: 7 - 252 days). Revision of the port was performed nine times in nine patients $(6.04 \%, 0.270$ of 1,000 catheter days). The procedures performed for port revision were as follows: four device exchanges over the wire $(2.2 \%, 0.098$ of 1,000 catheter days), four thrombolytics infusions (2.2\%, 0.098 of 1000 catheter days), and one saline flushing (0.55\%, 0.024 of 1,000 catheter days). A total of 25 complications (25/182, $13.7 \%, 0.616$ of 1,000 catheter days) occurred (Table 4 ). The incidence of periprocedural complications $(\leq 24 \mathrm{~h})$ was $1.65 \%$ (3/182, 0.074 of 1,000 catheter days). There were 22

\begin{tabular}{|lc}
\hline Table 2. Underlying Diseases $(\mathrm{N}=176)$ & Values \\
\hline Variables & 37 \\
\hline Lung cancer & 25 \\
\hline Hematologic malignancy & 24 \\
\hline Gastric cancer & 19 \\
\hline Colorectal cancer & 16 \\
\hline Breast cancer & 9 \\
\hline Pancreatic cancer & 7 \\
\hline Ovarian cancer & 5 \\
\hline Uterine cancer & 4 \\
\hline Esophageal cancer & 3 \\
\hline Cervical cancer & 23 \\
\hline Other malignancies & 4 \\
\hline Other disease & 2 \\
\hline Pneumonia & 1 \\
\hline Hypoxic brain damage & 1 \\
\hline Castleman disease & \\
\hline
\end{tabular}

\begin{tabular}{|lc}
\hline Table 3. Causes of Removal of Venous Access Ports (42 Events in 40 Patients) \\
\hline Variables & Values \\
\hline End of Treatment & 17 \\
\hline Catheter-related infection & 10 \\
\hline \multicolumn{1}{|c}{ Pocket infection } & 4 \\
\hline \multicolumn{1}{c}{ Catheter-related bloodstream infection } & 1 \\
\hline Central line-associated bloodstream infection & 5 \\
\hline Catheter occlusion & 2 \\
\hline Venous thrombosis & 1 \\
\hline Other reasons & 12 \\
\hline
\end{tabular}

post-procedural complications, including two early complications ( $\leq 30$ days: $2 / 182,1.10 \%, 0.049$ of 1,000 catheter days), and 20 late complications (> 30 days: 20/182, 10.99\%, 0.493 of 1,000 catheter days). In total, 10 of 182 patients (5.49\%, 0.246 of 1,000 catheter days) experienced catheterrelated infections, including six patients $(3.30 \%, 0.148$ of 1,000 catheter days) with bloodstream infections (five CLABSI and one CRBSI) and four patients $(2.20 \%, 0.099$ of 1,000 catheter days) with pocket infections. The total access site device interval of ports with catheter-related infections was 1,617 days (mean, 161.7 catheter days; range: 8 548 catheter days). Throughout the study period, 14 (7.69\%, 0.345 of 1,000 catheter days) minor complications and 11 $(6.04,0.271$ of 1,000 catheter days) major complications occurred in 182 TIVAPs. 


\begin{tabular}{|c|c|c|c|c|c|}
\hline Time & Grade & Type & Incidence & Event/1000 days & Treatment \\
\hline$\leq \mathbf{2 4 h}$ & Minor & Catheter occlusion & $3(1.65 \%)$ & 0.074 & $\begin{array}{c}\text { Thrombolysis }(n=2) \text {; Exchange over } \\
\text { the wire }(n=1)\end{array}$ \\
\hline \multirow{2}{*}{$\leq \mathbf{3 0}$ days } & Minor & Extravasation during transfusion & $1(0.55 \%)$ & 0.025 & Change port needle \\
\hline & Major & $\begin{array}{c}\text { Thrombosis in axillary and brachial } \\
\text { veins }\end{array}$ & $1(0.55 \%)$ & 0.025 & Removal, Anticoagulation \\
\hline \multirow{5}{*}{$>30$ days } & \multirow{3}{*}{ Minor } & Wound dehiscence & $2(1.10 \%)$ & 0.049 & $\operatorname{Removal}(\mathrm{n}=1) ;$ Skin repair $(\mathrm{n}=1)$ \\
\hline & & Catheter occlusion & $7(3.85 \%)$ & 0.172 & Saline flushing; Thrombolysis \\
\hline & & Catheter kinking & $1(0.55 \%)$ & 0.025 & Exchange over the wire \\
\hline & \multirow{2}{*}{ Major } & Catheter-related infection & $6(3.30 \%)$ & 0.148 & Removal, Antibiotics \\
\hline & & Pocket infection & $4(2.20 \%)$ & 0.099 & Removal, Antibiotics \\
\hline
\end{tabular}

\section{Discussion}

The conventional technique has several potential disadvantages associated with the creation of two incisions: difficulty in advancing the tunneling devices, risk of bleeding, pain at the tunneling site, and incorrect measurement of the catheter length. These problems can lead to catheter malfunction and venous thrombosis. Postprocedural problems include discomfort in the subcutaneous tunnel overlying the clavicle with neck movement or swallowing, and esthetic disadvantages. In patients with a low body mass index, it is difficult to create a subcutaneous tunnel in deep subcutaneous tissue (4-7).

To overcome the limitations of the conventional technique, a single-incision technique through the IJV, axillary vein, and subclavian vein has been used for TIVAP placement. The advantages of the single-incision technique include elimination of a second venotomy incision, reduction of bleeding risk, shortened procedure time, fewer infections related to the neck incision, esthetic advantages, and reduction of postoperative discomfort by avoiding the requirement for a neck dressing $(4-8,14)$. The limitations of the single-incision technique via IJV are limited subcutaneous tunnel length based on needle length, a potentially inaccessible IJV depending on the patient's anatomy or available needle length, and difficulty in advancing devices with a hairpin-turn shape (6).

Although the procedure time for the single-incision technique was not surveyed in the present study, the procedure time of the single-incision technique is generally similar to or less than that of the conventional technique, based on the authors' experience. Seo et al. (6) reported that the mean procedure time of TIVAP insertion using the single-incision technique was 13 min $39 \mathrm{~s}$, which was shorter than the procedure time reported in a previous study (generally 20 - $30 \mathrm{~min}$; maximum, $50 \mathrm{~min}$ ) (4). The main source of the shortened duration of the singleincision technique is the simpler procedure, compared to the conventional technique, due to the absence of subcutaneous tunneling, lack of requirement for IJV access, and lack of suturing at the vascular access site.

In this study, the overall complication rate was $13.74 \%$ (25/182). Among the total 25 complications, three occurred in the periprocedural early phase (1.65\%), two in the early phase (1.10\%), and 20 in the late phase (10.99\%). There were no major complications that resulted in prolonged hospitalization, permanent sequelae, or death $(9,10)$. Considering the low periprocedural complication rate and the absence of any major complications, the single-incision technique is similar in safety to the conventional technique. The incidence of catheter-related infection was $5.49 \%$ in this study; the incidences of catheter-related infection were reportedly $1.7 \%-8.8 \%$ in other studies $(8,15-$ $20)$. In the present study, catheter-related infections were noted in the late phase of the follow-up period, which might have been due to patient factors (e.g., weakened immune status of patients with malignant disease, ongoing chemotherapy, and comorbidities), rather than differences between the two techniques with respect to implantation of the TIVAP $(11,20,21)$. The incidence of pocket infection in this study was $2.20 \%$, which was comparable to the rate reported in a previous study (range: $2.6 \%$ - 9\%) (6).

The right IJV is the preferred access site for the insertion of central venous catheter because of its high technical success rate and low complication rate (22). However, in most oncology patients, thrombosis and central vein stenosis are occult in nature and respond well to anticoagulation and/or catheter removal (4). In the present study, symptomatic venous thrombosis occurred in one patient $(0.55 \%)$, which was the only major complication observed within 30 days after the procedure. The incidence of thrombosis was lower than that reported in previous studies (range: $0.67 \%-8.4 \%$ ); it was also lower than the threshold suggested (6\%) in quality improvement guidelines (8, $9,23)$.

The limitations of this study were as follows. First, 
due to the retrospective nature of this study, complication rates could have been documented in association with the type of port, or the use of prophylactic antibiotics. Some patients were excluded because they had been lost to follow-up or transferred to other hospitals, which may have resulted in under- or overestimation of the complication rates. Second, no comparisons of the outcomes and complications were performed between the singleincision and conventional techniques. To fully document the procedure time, outcomes, and complications, further randomized prospective research studies comparing the two techniques are required. Third, patient satisfaction was not analyzed in terms of post-procedural pain, cosmetic issues, or any discomfort related to the procedures. Following the use of the single-incision technique, no patients reported discomfort over the clavicle or lower neck, which is a frequent complaint of patients who undergo TIVAP using the conventional technique.

In conclusion, the single-incision technique for TIVAP placement via the axillary vein is a feasible and safe procedure with high technical and clinical success rates, as well as a low complication rate. Placement of the TIVAP insertion by the single-incision technique may be a good alternative to the conventional method.

\section{Footnotes}

Authors' Contributions: Jae Hyun Kwon designed this paper and performed critical revision of the manuscript. Jae Hyun Kwon and Seo Jin Jang performed data collection and wrote the manuscript. Yoon Ki Cha and DoYeun Kim performed study supervision and critical revision of the manuscript for intellectual content. All authors read and approved the final manuscript.

Conflict of Interests: The authors declare no conflict of interests.

Ethical Approval: This study was conducted in accordance with the Declaration of Helsinki and approval from the Ethics Committee of Dongguk University Ilsan Hospital (No. DUIH 2017-07-008-005).

Funding/Support: The author(s) received no financial support for the research, authorship, and/or publication of this article.

\section{References}

1. Ashton D, Variyam D, Hernandez JA, Pimpalwar S, Hayatghaibi S, Kukreja K. Single-Incision Versus Conventional Technique for Tunneled Central Line Placement in Children. Cardiovasc Intervent Radiol. 2017;40(10):1552-8. doi: 10.1007/s00270-017-1682-5. [PubMed: 28488105].

2. Nakamura T, Sasaki J, Asari Y, Sato T, Torii S, Watanabe M. Complications after implantation of subcutaneous central venous ports (PowerPort()). Ann Med Surg (Lond). 2017;17:1-6. doi: 10.1016/j.amsu.2017.03.014. [PubMed: 28348733]. [PubMed Central: PMC5358939].
3. Kock HJ, Pietsch M, Krause U, Wilke H, Eigler FW. Implantable vascular access systems: experience in 1500 patients with totally implanted central venous port systems. World J Surg. 1998;22(1):12-6.

4. Glenn BJ. Single-incision method for the placement of an implantable chest port or a tunneled catheter.JVasc Interv Radiol.2007;18(1 Pt1):13740. doi: 10.1016/j.jvir.2006.09.001. [PubMed:17296715].

5. Contractor SG, Phatak TD, Klyde D, Gonzales S, Sadowski S, Bhagat N. Single-incision technique for tunneled central venous access. $J$ Vasc Interv Radiol. 2009;20(8):1052-8. doi: 10.1016/j.jvir.2009.04.065. [PubMed: 19647183].

6. Seo TS, Song MG, Kang EY, Lee CH, Yong HS, Doo K. A singleincision technique for placement of implantable venous access ports via the axillary vein. I Vasc Interv Radiol. 2014;25(9):1439-46. doi: 10.1016/j.jvir.2013.12.571. [PubMed: 24613268].

7. Charles HW, Miguel T, Kovacs S, Gohari A, Arampulikan J, McCann JW. Chest port placement with use of the single-incision insertion technique. J Vasc Interv Radiol. 2009;20(11):1464-9. doi: 10.1016/j.jvir.2009.07.035. [PubMed: 19875065].

8. Hong S, Seo TS, Song MG, Seol HY, Suh SI, Ryoo IS. Clinical outcomes of totally implantable venous access port placement via the axillary vein in patients with head and neck malignancy. I Vasc Access. 2019;20(2):134-9. doi: 10.1177/1129729818781270. [PubMed: 29923460].

9. Dariushnia SR, Wallace MJ, Siddiqi NH, Towbin RB, Wojak JC, Kundu S, et al. Quality improvement guidelines for central venous access. J Vasc Interv Radiol. 2010;21(7):976-81. doi: 10.1016/j.jvir.2010.03.006. [PubMed: 20610180].

10. Silberzweig JE, Sacks D, Khorsandi AS, Bakal CW; Society of Interventional Radiology Technology Assessment Committee. Reporting standards for central venous access. J Vasc Interv Radiol. 2003;14(9 Pt 2):S443-52. doi: 10.1097/01.rvi.0000094617.61428.bc. [PubMed: 14514860].

11. O'Grady NP, Alexander M, Dellinger EP, Gerberding JL, Heard SO, Maki DG, et al. Guidelines for the prevention of intravascular catheter-related infections. Centers for Disease Control and Prevention. MMWR. Recommendations and reports: Morbidity and mortality weekly report. Recommendations and reports. 2002;51(RR-10):1-29.

12. Bell T, O'Grady NP. Prevention of Central Line-Associated Bloodstream Infections. Infect Dis Clin North Am. 2017;31(3):551-9. doi: 10.1016/j.idc.2017.05.007. [PubMed: 28687213]. [PubMed Central: PMC5666696].

13. Mermel LA, Allon M, Bouza E, Craven DE, Flynn P, O'Grady NP, et al Clinical practice guidelines for the diagnosis and management of intravascular catheter-related infection: 2009 Update by the Infectious Diseases Society of America. Clin Infect Dis. 2009;49(1):1-45. doi: 10.1086/599376. [PubMed: 19489710]. [PubMed Central: PMC4039170].

14. Seo TS, Song MG, Kim JS, Choi CW, Seo JH, Oh SC, et al. Long-term clinical outcomes of the single-incision technique for implantation of implantable venous access ports via the axillary vein. $J$ Vasc Access 2017;18(4):345-51. doi: 10.5301/jva.5000751. [PubMed: 28665466].

15. Lorch H, Zwaan M, Kagel C, Weiss HD. Central venous access ports placed by interventional radiologists: experience with 125 consecutive patients. Cardiovasc Intervent Radiol. 2001;24(3):180-4. doi: 10.1007/s002700001721. [PubMed: 11443406].

16. Gebauer B, El-Sheik M, Vogt M, Wagner HJ. Combined ultrasound and fluoroscopy guided port catheter implantation-high success and low complication rate. Eur J Radiol. 2009;69(3):517-22. doi: 10.1016/j.ejrad.2007.10.018. [PubMed: 19340958].

17. Hsieh CC, Weng HH, Huang WS, Wang WK, Kao CL, Lu MS, et al. Analysis of risk factors for central venous port failure in cancer patients. World J Gastroenterol. 2009;15(37):4709-14. doi: 10.3748/wjg.15.4709. [PubMed: 19787834]. [PubMed Central: PMC2754519].

18. Çil BE, Canyigit M, Peynircioglu B, Hazirolan T, Çarkaci S, Çekirge S, et al. Subcutaneous venous port implantation in adult patients: a single center experience. Diagn Interven Radiol. 2006;12(2):93.

19. Schwarz RE, Groeger JS, Coit DG. Subcutaneously implanted central venous access devices in cancer patients. Cancer. 1997;79(8):1635-40. doi: 10.1002/(sici)1097-0142(19970415)79:8<1635::aid-cncr30>3.0.co;2 
20. Teichgraber UK, Kausche S, Nagel SN, Gebauer B. Outcome analysis in 3,160 implantations of radiologically guided placements of totally implantable central venous port systems. Eur Radiol. 2011;21(6):122432. doi: 10.1007/s00330-010-2045-7. [PubMed: 21207035].

21. Shim J, Seo TS, Song MG, Cha IH, Kim JS, Choi CW, et al. Incidence and risk factors of infectious complications related to implantable venous-access ports. Korean J Radiol. 2014;15(4):494-500. doi: 10.3348/kjr.2014.15.4.494. [PubMed: 25053910]. [PubMed Central: PMC4105813].
22. Funaki B, Szymski GX, Hackworth CA, Rosenblum JD, Burke R, Chang $\mathrm{T}$, et al. Radiologic placement of subcutaneous infusion chest ports for long-term central venous access. AJR Am J Roentgenol. 1997;169(5):1431-4. doi: 10.2214/ajr.169.5.9353475. [PubMed: 9353475].

23. Caers J, Fontaine C, Vinh-Hung V, De Mey J, Ponnet G, Oost C, et al. Catheter tip position as a risk factor for thrombosis associated with the use of subcutaneous infusion ports. Support Care Cancer. 2005;13(5):325-31. doi: 10.1007/s00520-004-0723-1. [PubMed: 15538639]. 